\title{
Nonlinear MHD simulations of Quiescent H-mode plasmas in DIII-D
}

\author{
F. Liu ${ }^{1}$, G.T.A. Huijsmans ${ }^{1}$, A. Loarte ${ }^{1}$, A. M. Garofalo ${ }^{2}$, \\ W. M. Solomon ${ }^{3}$, P. B. Snyder ${ }^{2}$, M. Hoelzl ${ }^{4}$ \\ ${ }^{1}$ ITER Organization, Route de Vinon sur Verdon, 13067 Saint Paul Lez Durance, France \\ ${ }^{2}$ General Atomics, P.O. Box 85608, San Diego, California 92186-5608, USA \\ ${ }^{3}$ Princeton Plasma Physics Laboratory, Princeton, New Jersey 08543-0451, USA \\ ${ }^{4}$ Max Planck Institute for Plasma Physics, 85748 Garching, Germany
}

In the Quiescent H-mode (QH-mode) regime, the edge harmonic oscillation (EHO), thought to be a saturated kink-peeling mode (KPM) driven unstable by current and rotation, is found in experiment to provide sufficient stationary edge particle transport to avoid the periodic expulsion of particles and energy by edge localized modes (ELMs). In this paper, both linear and nonlinear MHD modelling of QH-mode plasmas from the DIII-D tokamak have been investigated to understand the mechanism leading to the appearance of the EHO in QH-mode plasmas. For the first time nonlinear MHD simulations with low-n modes both with ideal wall and resistive wall boundary conditions have been carried out with 3-D non-linear MHD code JOREK. The results show, in agreement with the original conjectures, that, in the nonlinear phase, kink peeling modes are the main unstable modes in QH-mode plasmas of DIII$\mathrm{D}$ and that the kink-peeling modes saturate non-linearly leading to a 3-D stationary state. The characteristics of the kink-peeling modes, in terms of mode structure and associated decrease of the edge plasma density associated with them, are in good agreement with experimental measurements of the EHO in DIII-D. The effect of plasma resistivity, the role of plasma parallel rotation as well as the effect of the conductivity of the vacuum vessel wall on the destabilization and saturation of kink-peeling modes have been evaluated for experimental QH-mode plasma conditions in DIII-D.

\section{Introduction}

The good energy confinement in the H-mode makes it the preferred operation regime for high performance operation in present tokamak devices and the reference operational scenario to achieve high fusion gain in ITER. However, edge localized modes (ELMs) driven by large pressure and current gradients in the H-mode edge transport barrier can produce high transient power and particle loads to the plasma facing components (PFCs), which can lead to increased erosion of the divertor and reduced divertor target lifetime in ITER [1,2]. On the other hand, the time-averaged edge particle transport provided by ELMs is required to provide impurity control both for intrinsic and extrinsic impurities as well as for the prevention of helium ash accumulation in burning plasma [3]. Due to the detrimental effects of ELMs on the plasma facing components' lifetime, there is a large ongoing effort on ELM control in both the experimental and the theoretical/modelling areas. In ITER, two methods 
for ELM control are foreseen in the baseline: increasing the ELM frequency by pellet injection [4], and stabilisation or mitigation of ELMs by the application of 3-D edge magnetic field perturbations with external coils [5]. The use of vertical kicks to trigger ELMs is also being investigated as an option for ELM control to provide tungsten impurity control in the initial phases of ITER operation in which the plasma current for $\mathrm{H}$-mode discharges will typically be 7.5 MA [6]. The extrapolation of all the techniques for active ELM control to ITER is subject to uncertainties, as their physics basis is not known with sufficient precision, and there exist issues regarding their compatibility with particular scenario/hardware requirements in ITER (compatibility with radiative divertor operation, required fuel throughput, etc.). For this reason, research on confinement regimes which can reach high energy confinement levels without ELMs remains a priority for ITER. In this respect, the QH-mode regime, originally developed at the DIII-D tokamak [7, 8] and investigated in other current devices [9-11], has been found to provide high energy confinement without transient energy fluxes to PFCs associated with ELMs. In DIII-D, this operational regime has recently been extended towards conditions suitable for ITER operation such as low torque input [12] and high normalized density operation [13] thus overcoming two important restrictions for its suitability for ITER. In the QH-mode, an edge harmonic oscillation (EHO) is found to provide stationary edge particle transport that replaces the periodic expulsion of particles and energy by ELMs. The EHO is conjectured to be a saturated external kink/peeling mode driven unstable by edge current and rotation. The increased pedestal transport associated with this MHD instability appears to be sufficient to maintain the edge pressure gradient near but below the ELM instability boundary [14] thus avoiding the triggering of ELMs. Although the reference scenario for ITER Q = 10 operation is based on the ELM-controlled Type I ELMy H-mode (with 3-D edge magnetic field perturbations or controlled triggering of ELMs by pellets), for the reasons mentioned, it is of interest for ITER to assess whether the QH-mode could be a viable alternative for such scenario in ITER. This requires the understanding of the nonlinear MHD physics mechanisms leading to the EHO experimental behaviour found in the QH-mode experiments, including the role of plasma rotation in this observed behaviour, as a first step towards determining whether such physics mechanisms would be at work in ITER plasmas and thus whether the QH-mode can be considered as an alternative to the controlled Type I ELMy H-mode for ITER high Q operation.

In this paper both linear MHD stability limits and the nonlinear evolution of DIII-D QHmode plasmas have been investigated. In particular and for the first time, nonlinear MHD simulations of DIII-D QH-mode plasmas have been carried out with the nonlinear MHD code 
JOREK [15-16]. The physics characteristics of the non-linearly saturated kink-peeling mode, conjectured to be associated in the experiment with the EHO, will be presented and compared with experimental observations of the EHO to demonstrate the validity of the conjecture. Finally, the influence of parallel rotation as well as of the effect of the conductivity of the vacuum vessel wall on the destabilization and saturation of kink-peeling modes for DIII-D QH-mode plasma will be discussed.

In this paper we consider two DIII-D QH-mode discharges: one is chosen because it is representative of what could be possible in ITER with the available input torque and externally imposed 3-D fields (\#145117) and the other because it displays a robust QH-mode behaviour although its experimental requirements would not be available in ITER (\#153440). \#145117 is a discharge with $\mathrm{I}_{\mathrm{p}}=1.1 \mathrm{MA}, \mathrm{B}_{\mathrm{t}}=1.9 \mathrm{~T}$, counter beam neutral injection at first and then with increasing co-injection to a relevant ITER-like co-torque level and with 3-D nonaxisymmetric (NA) magnetic field perturbation applied. \#153440 is a discharge with $\mathrm{I}_{\mathrm{p}}$ $=1.4 \mathrm{MA}, \mathrm{B}_{\mathrm{t}}=1.9 \mathrm{~T}$ and strong counter neutral beam injection throughout and without NA fields. The equilibria used in the analysis presented in this paper have been obtained from reconstructions with the EFIT code [17] using the magnetic and profile data for these discharges.
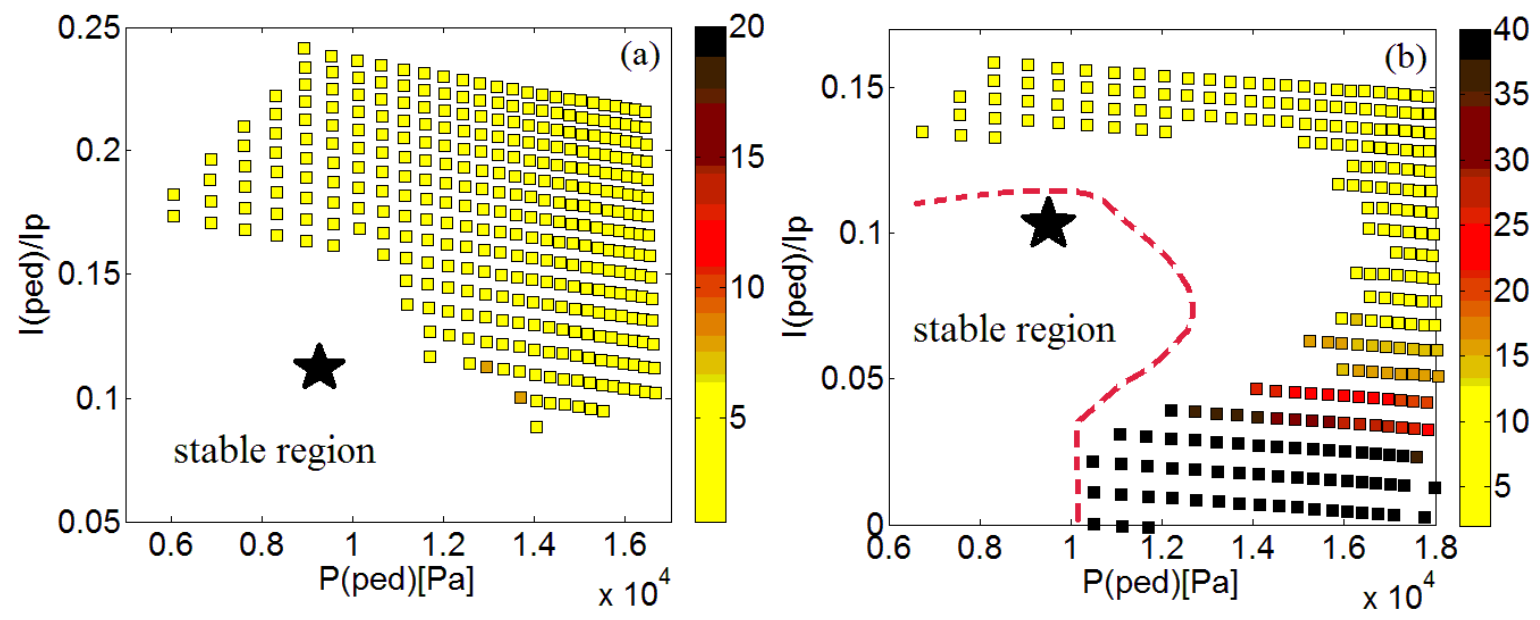

Figure 1. (a) Peeling-ballooning mode stability diagram for \#145117. (b) Peeling-ballooning mode stability diagram for \#153440. In both plots, the stars represent the equilibrium of the discharges, peeling-ballooning modes are scanned for toroidal number $\mathrm{n} \in[1,40]$, squares are unstable modes colored by the most unstable toroidal number $\mathrm{n} \in[1,40]$, the dashed red line in $(b)$ is stable boundary when $\mathrm{J}_{\text {sep }} / \mathrm{J}_{0}=0.4$ for \#153440.

\section{Linear MHD stability analysis}

We have started the analysis of these discharges by studying the ideal MHD stability limits with respect to peeling-ballooning modes at the pedestal of these QH-mode plasmas with the linear ideal MHD code MISHKA-1 [18]. The edge stability diagrams for these discharges are 
constructed by varying the pedestal pressure gradient and the pedestal current density independently. For each equilibrium, the growth rate is calculated for modes with toroidal mode numbers between $n=1$ to $n=40$. In the stability calculations, the plasma is assumed to be surrounded by a vacuum and an ideally conducting wall at the minor radius position $r_{w a l l}$ $=1.15 \mathrm{a}$, where $\mathrm{a}=0.68 \mathrm{~m}$ is the minus radius. The stability diagram for the ideal MHD peelingballooning stability of the edge plasma for shot \#145117 is shown in Figure 1(a). The experimental equilibrium, represented by the star, is in the stable region but close to the corner of the kink/peeling and ballooning stability boundary, in agreement with earlier results for DIII-D QH-mode plasmas using the ELITE code [19]. The peeling-ballooning diagram for shot \#153440 is shown in Figure 1(b). This plasma lies in a stable region but closer to the kink/peeling stability boundary than to the ballooning one. The presence of a large bootstrap current in the pedestal of these plasmas is the reason behind their closeness to the kink/peeling boundary.
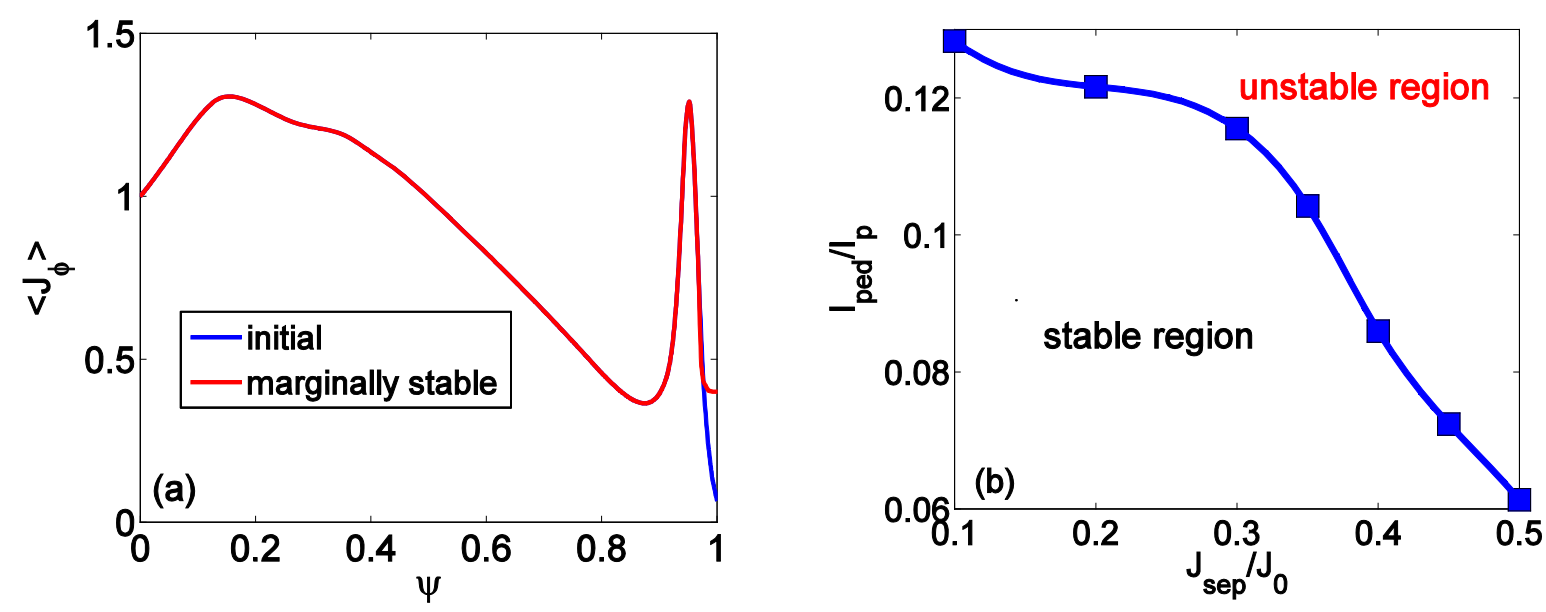

Figure 2. (a) The flux surface averaged current density profile (blue) of $\# 153440$ as a function of the normalized poloidal flux, including the current density profile that is the marginally stable to kinkpeeling modes (red). (b) The kink-peeling stability limit in terms of the pedestal current normalized to the total plasma current as a function of the normalized separatrix current density.

The kink/peeling stability boundary is found to depend sensitively on the value of the current density at the separatrix. Figure 2(b) shows the dependence of the kink-peeling limit as a function of the separatrix current density $\left(\mathrm{J}_{\text {sep }}\right)$ normalised to the central current density $\left(\mathrm{J}_{0}\right)$. Increasing the edge current density to $\mathrm{J}_{\text {sep }} / \mathrm{J}_{0}=0.4$ lowers the kink-peeling limit to the values obtained from the experimental equilibrium reconstruction for shot \#153440. The ballooning stability boundary, on the other hand, is not affected by the separatrix current density. Thus a small change to the current density profile in the edge region, as shown in Figure 2(a), is 
sufficient to bring the experimental equilibrium to reach the ideal MHD kink-peeling stability boundary.

\section{Nonlinear MHD simulations of DIII-D QH-mode plasmas}

\subsection{The non-linear MHD code JOREK}

The JOREK code [15-16] is a 3-D non-linear MHD code, developed with the aim of studying the non-linear evolution of MHD instabilities such as ELMs, tearing modes, kink/peeling and ballooning modes, in full toroidal X-point geometry including the separatrix, open and closed field lines. Both the full MHD model and reduced MHD models are available in JOREK. For the study presented here, the reduced MHD model, detailed below, has been applied. The magnetic field $\vec{B}$ and the velocity $\vec{v}$ are represented using the poloidal flux $\psi$ and the electric potential $\Phi$ and the parallel velocity $v_{/ /}$with the following ansatz:

$\boldsymbol{B}=\boldsymbol{B}_{\phi}+\boldsymbol{B}_{p}=\frac{F_{0}}{R} \boldsymbol{e}_{\boldsymbol{\phi}}+\frac{1}{R} \nabla \psi \times \boldsymbol{e}_{\boldsymbol{\phi}}$,

$\boldsymbol{v}=\boldsymbol{v}_{/ /}+\boldsymbol{v}_{\perp}=v_{/ /} \boldsymbol{B}+R \boldsymbol{e}_{\boldsymbol{\phi}} \times \nabla \Phi$

where $R$ is the major radius in toroidal geometry, $\boldsymbol{e}_{\boldsymbol{\phi}}$ is the toroidal unit vector and $F_{0}=B_{0} R_{0}$, with the magnetic field strength $B_{0}$ at the magnetic axis $R=R_{0}$. Substituting Equation (1) and (2) into the visco-resistive MHD equations including diffusive particle and heat transport leads to the reduced MHD model, to be solved for mass density $\rho$, temperature $T$, the electric potential $\Phi$, the parallel velocity and the poloidal flux:

$\frac{\partial \rho}{\partial t}=-\nabla \cdot(\rho \boldsymbol{v})+\nabla \cdot\left(D_{\perp} \nabla_{\perp} \rho\right)+S_{\rho}$,

$\rho \frac{\partial T}{\partial t}=-\rho \boldsymbol{v} \cdot \nabla T-(\gamma-1) \rho T \nabla \cdot \boldsymbol{v}+\nabla \cdot\left(\kappa_{\perp} \nabla_{\perp}+\kappa_{/ /} \nabla_{/ /} T\right)+S_{T}$,

$\boldsymbol{e}_{\boldsymbol{\phi}} \cdot \nabla \times\left(\rho \frac{\partial \boldsymbol{v}}{\partial t}=-\rho(\boldsymbol{v}-\nabla) \boldsymbol{v}-\nabla(\rho T)+\boldsymbol{J} \times \boldsymbol{B}+\mu \Delta \boldsymbol{v}\right)$,

$\boldsymbol{B} \cdot\left(\rho \frac{\partial \boldsymbol{v}}{\partial t}=-\rho(\boldsymbol{v} \cdot \nabla) \boldsymbol{v}-\nabla(\rho T)+\boldsymbol{J} \times \boldsymbol{B}+\mu \Delta \boldsymbol{v}\right)$,

$\frac{1}{R^{2}} \frac{\partial \psi}{\partial t}=\eta(T) \nabla \cdot\left(\frac{1}{R^{2}} \nabla_{\perp} \psi\right)-\boldsymbol{B} \cdot \nabla \Phi$,

where $D_{\perp}, \kappa_{\perp}$ and $\kappa_{/ /}$are the perpendicular particle diffusion and the perpendicular and temperature dependent parallel heat conductivity. $S_{\rho}$ and $S_{T}$ are the particle and heat source, respectively. $\eta=\eta_{0}\left(T / T_{0}\right)^{-3 / 2}$ is the temperature dependent plasma resistivity, $\mu$ is the viscosity. $\boldsymbol{J}$ is the toroidal current density and $\gamma$ is the ratio of the specific heats. 
The normalization used in the equations is based on the magnetic permeability $\mu_{0}$ and the mass density $\rho_{0}$ on axis. The diffusive parameters are normalized as $\eta_{J}=\eta_{S I} \sqrt{\rho_{0} / \mu_{0}}$, $\mu=\mu_{S I} \sqrt{\mu_{0} / \rho_{0}}, D_{\perp}=D_{\perp S I} \sqrt{\mu_{0} \rho_{0}}, \kappa_{\perp}=\kappa_{\perp S I} \sqrt{\mu_{0} / \rho_{0}}$, where the SI subscript refers to the values of the respective coefficients in the SI unit system.

The boundary condition on the surfaces parallel to the magnetic field is usually taken to be an ideally conducting wall in JOREK. Coupling of JOREK to the STARWALL code [20] allows a resistive wall boundary condition to be modelled (see Section 5 for more details). On open field lines, the parallel component of the velocity is set to the local sound speed in the outgoing direction (Mach-one sheath boundary condition). The boundary condition on the parallel energy flux is described by a sheath transmission factor relating the convective and conductive energy fluxes [21].

In the JOREK code, both physics variables and the $(\mathrm{R}, \mathrm{Z})$ coordinates of the poloidal plane are described by bi-cubic $\mathrm{C}^{1}$ Bezier finite elements [22], giving to a continuous representation of the variables and their derivatives. The toroidal direction is discretized using a real Fourier series. The time evolution scheme is fully implicit, advancing all variables in a single step using a Crank-Nicholson or Gears scheme. The resulting large sparse matrix is iteratively solved with the GMRES method. The diagonal block matrices corresponding to the individual toroidal harmonics are applied as an effective preconditioner, which are solved using the direct parallel sparse matrix solver PaStiX [23].

\subsection{Non-linear MHD simulation of QH-mode plasmas}

Nonlinear MHD simulations of DIII-D shot \#145117 performed with the JOREK code assuming ideal wall boundary conditions have been carried out. The influence of 3-D NA fields, which are applied in the experiment for this discharge, is not modelled in the study presented here. In a first step, the initial 2-D static equilibrium obtained from a kinetic EFIT fit is evolved in JOREK on a finite element grid aligned to the poloidal flux. A typical grid size in our studies includes 7-10 thousand bi-cubic finite elements in the poloidal plane and up to 32 toroidal planes. The initial static equilibrium for the $n=0$ toroidal harmonic obtained from EFIT is evolved in time with JOREK in order to obtain a new stationary equilibrium with consistent equilibrium flows. In this evolution the transport coefficients in JOREK are chosen such as to keep the density and pressure profile approximately constant in time and similar to the experimental ones from EFIT. In the pedestal, the gradients are preserved by choosing a local minimum in the profile of the diffusion coefficients applied in the JOREK 
simulations. For these simulations the perpendicular particle diffusion and perpendicular and parallel heat conductivity are chosen as $D_{\perp}=1 \times 10^{-6}, \kappa_{\perp}=1 \times 10^{-6}$ and $\kappa_{/ /}=500$ (in normalised units). The pedestal diffusivities are chosen as $D_{\perp p e d}=0.08 D_{\perp}$ and $\kappa_{\perp p e d}=0.25 \kappa_{\perp}$ to keep the original pedestal profiles constant over time (in SI units $\kappa_{\perp}=2 \mathrm{~m}^{2} \mathrm{~s}^{-1}$ ). A heating and a particle source are applied to keep the total energy and particle content in the stationary state without MHD activity.

After the JOREK equilibrium is obtained, simulations are carried out including higher toroidal harmonics. Figure 3 shows the time evolution of the magnetic energy perturbation for the simulation of shot \#145117 including toroidal harmonics $n=1-5$. The JOREK simulations show that there is an initial exponentially growing phase of MHD modes, as shown in Figure 3(a). This linear growth phase, which lasts from $0.8 \mathrm{~ms}$ to $1.3 \mathrm{~ms}$ is dominated by the medium-n $(n=5)$ harmonics. However, due to nonlinear coupling between $n=5,4$ and 3 , the growth of the $n=1,2$ harmonics is also excited later in the time evolution (at $1.25 \mathrm{~s}$ for $\mathrm{n}=1$ and $1.35 \mathrm{~s}$ for $\mathrm{n}=2$ ) with non-linear growth rates about 10 times larger than their linear growth rates earlier in the time evolution. After this initial growth phase a nonlinear phase starts, shown in detail in Figure $3(b)$, where the amplitude of the MHD perturbation saturates into a 3-D stationary state. The dominant mode number of the MHD perturbation changes from $n=5$ in the linear phase down to a predominantly $n=1$ perturbation in the stationary state, with the $n=5$ harmonic decaying to the smallest amplitude in the stationary state.
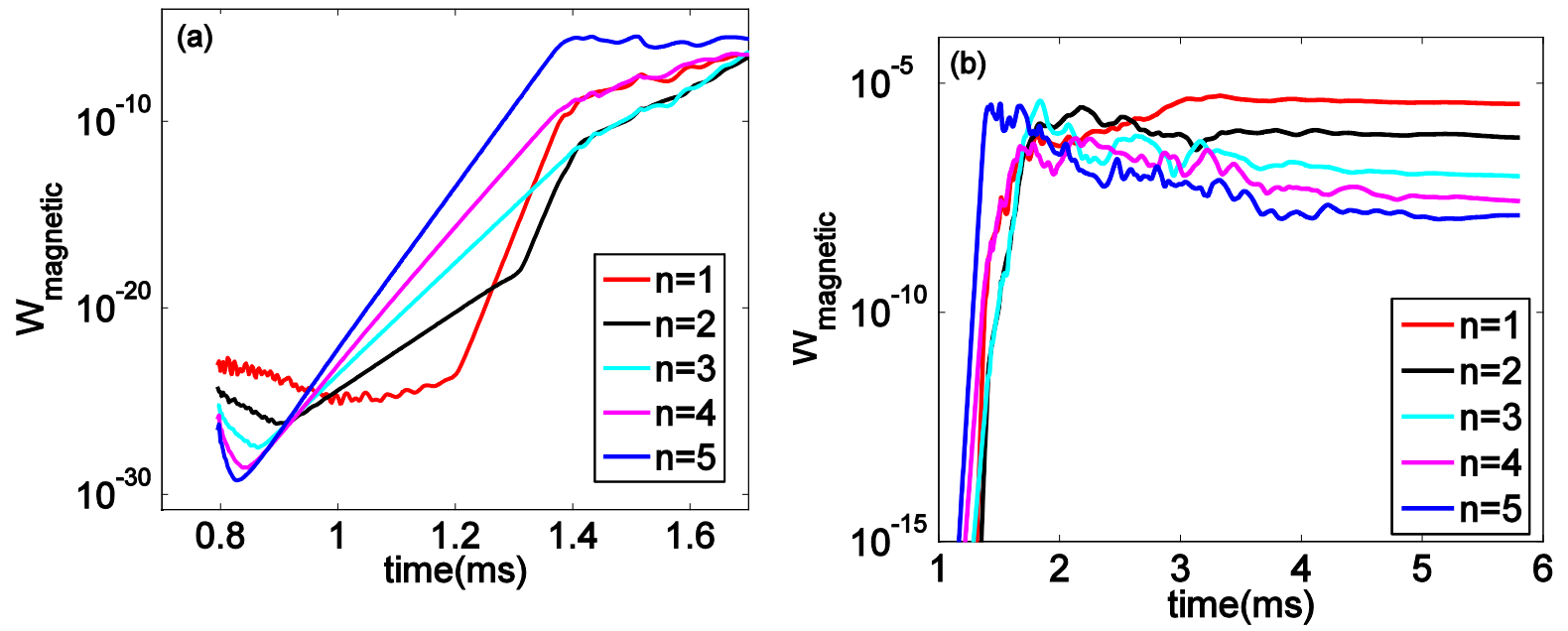

Figure 3. Time evolution of the perturbed magnetic energy of each harmonic for $n=1-5$ kink peeling mode for the JOREK simulations of shot \#145117. (a) Energy evolution in the linear phase, time from $0.8 \mathrm{~ms}$ to $1.7 \mathrm{~ms}$. (b) Energy evolution at the saturated phase, time from $1 \mathrm{~ms}$ to $6 \mathrm{~ms}$. 
Figure 4(a) shows a contour plot of the flux perturbation during the saturated state in Figure 3 (b) (including toroidal mode numbers $n=1-5$ ) with the typical mode structure of a nonlinearly saturated kink/peeling mode (KPM) [24] localised near the separatrix. Figure 4(b) shows the 3-D helical structure of the density on a flux surface corresponding to the unperturbed separatrix for the saturated $n=1-5$ KPM.
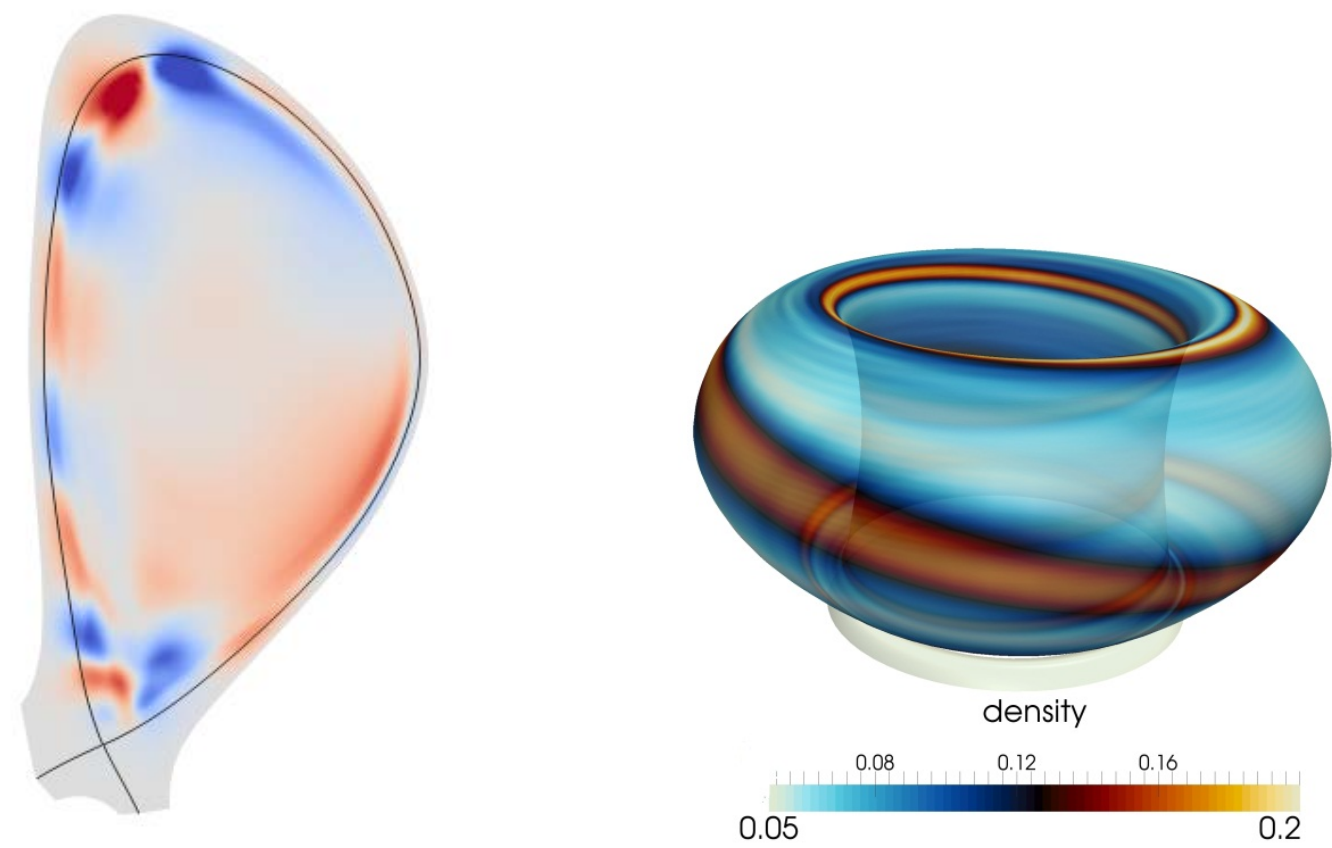

Figure 4. (a) Contour plot of the poloidal flux perturbation of the saturated $n=1-5$ KPM in the poloidal plane for the JOREK simulations of shot \#145117. (b) 3-D helical structure of the density perturbation for a saturated n=1-5 KPM at separatrix for the JOREK simulations of shot \#145117.

In these simulations the saturated KPM rotates in the counter clockwise poloidal direction due to the $E \times B$ velocity with a frequency about $1.6 \mathrm{k} \mathrm{Hz}$. The average $(n=0)$ flows are significantly increased by the presence of the KPM. The $\mathrm{E} \times \mathrm{B}$ flows are increased by a factor of 4, likely driven by the Maxwell stress (due to the magnetic perturbation of the instability) while the average parallel flows are increased by a factor of 2 . It should be noted that these simulations do not include diamagnetic, neoclassical or toroidal rotation effects. The rotating KPM leads to an oscillation of the plasma at the boundary which corresponds to a displacement of $1.5 \mathrm{~cm}$ at the outer mid-plane. 

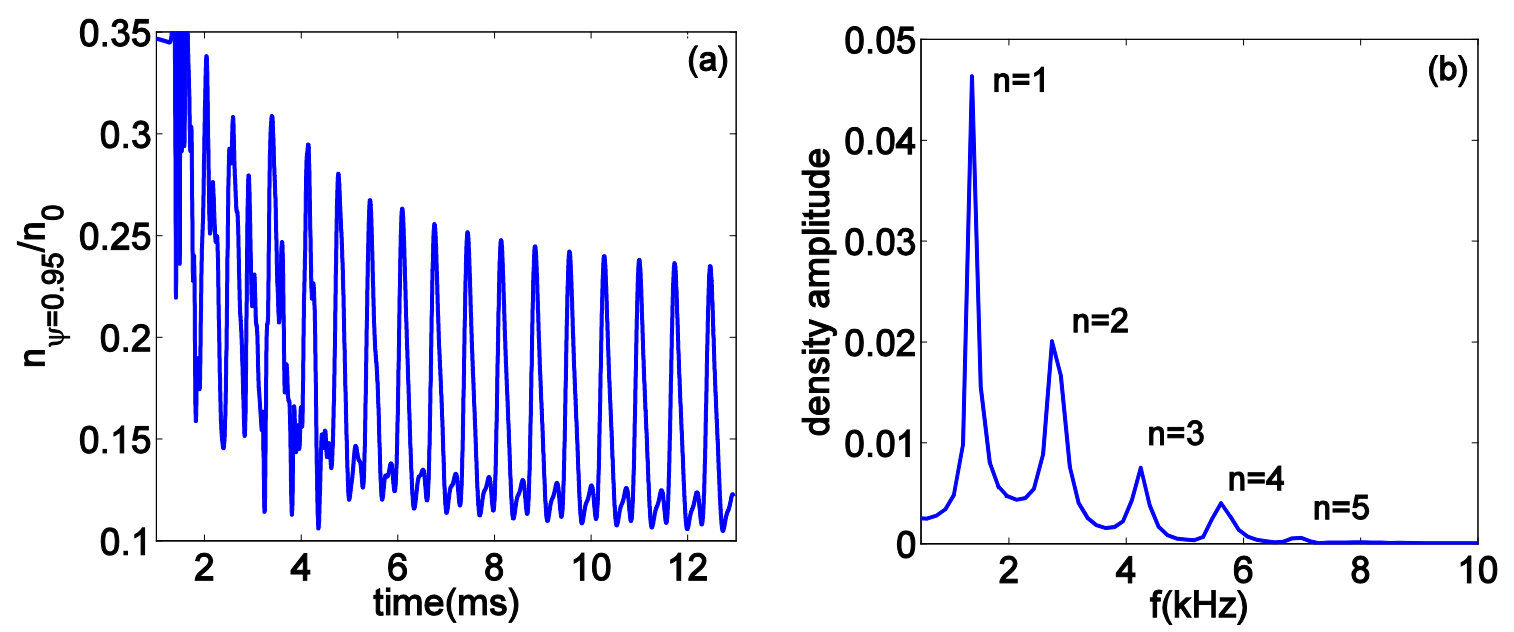

Figure 5. (a) Time evolution of the density (normalized to the central density) at normalized $\psi=0.95$ in the outer mid-plane for the JOREK simulations of shot \#145117. (b) Density frequency spectrum at normalized $\psi=0.95$ in outer mid-plane for the JOREK simulations of shot \#145117.

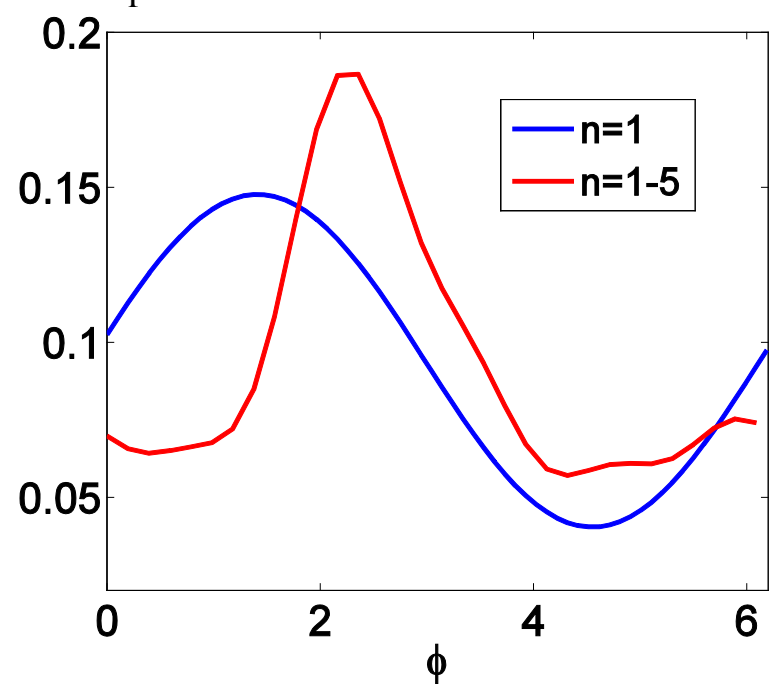

Figure 6. The amplitude of the density perturbation as a function of the toroidal angle $\phi$ at $\psi=0.95$ for $\mathrm{n}=1$ (blue curve) and n=1-5(red curve) for the JOREK simulations of shot \#145117.
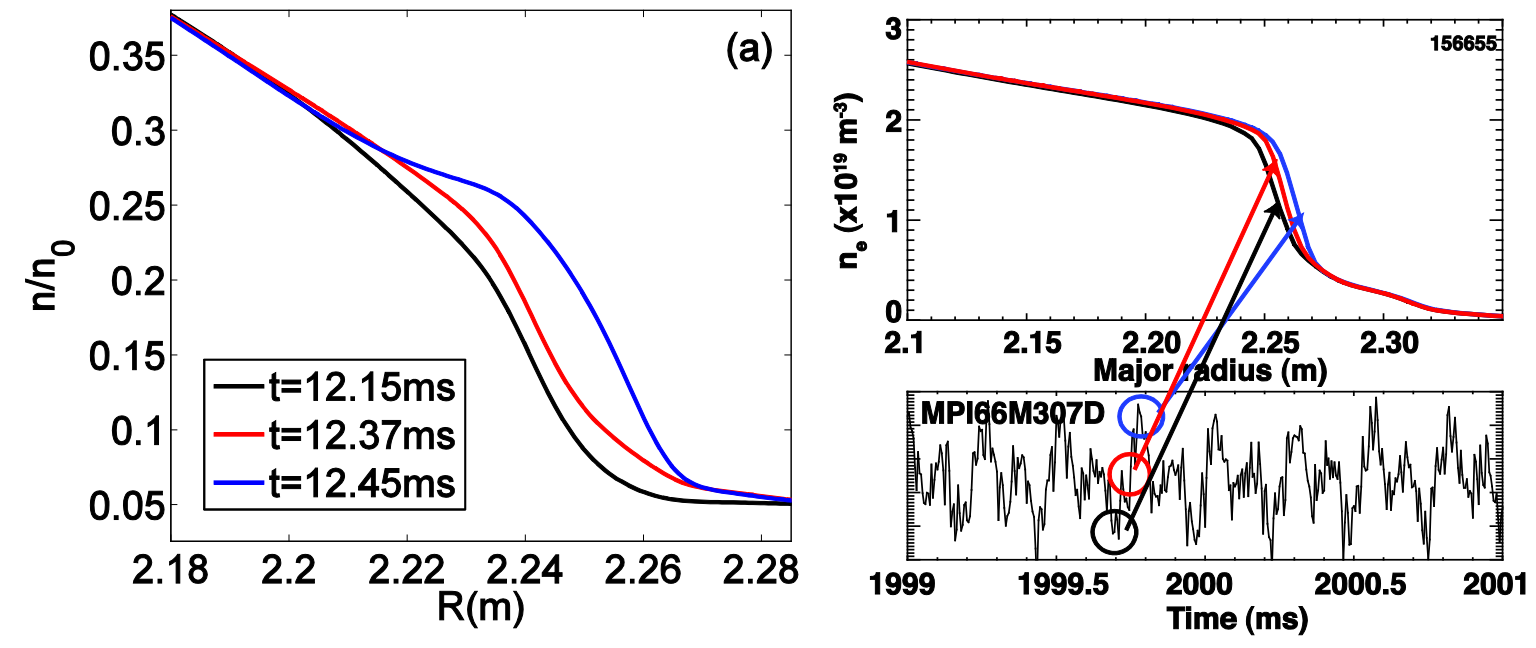
Figure 7. (a) Density profiles at 3 different times during the oscillation at the outboard mid-plane from the JOREK simulation of shot \#145117. (b) Density profiles at 3 different times at the outboard mid-plane in experimental measurement for DIII-D \#156655 [28].

The time evolution of the local density at normalized $\psi=0.95$ at the outer mid-plane for the $\mathrm{n}=1-5 \mathrm{KPM}$ is shown in Figure 5(a). The corresponding frequency spectrum of the density perturbation is plotted in Figure $5(b)$. The spectrum shows 5 equally spaced frequencies corresponding to the 5 toroidal harmonics used in the simulations. This indicates a coupling of the toroidal harmonics to form a, rotating, toroidally localized structure (as shown in Figure 4(b)). The non-sinusoidal behavior found in the simulations of the KPM mode, which is due to its toroidal localization due to coupling of harmonics, is in good agreement with the observations of the EHO typically present in DIII-D QH-mode experiments [12, 25, 26]. The amount of toroidal localization caused by harmonic coupling is illustrated in Figure 6 which shows the density as a function of the toroidal angle at the outer mid-plane at a normalized flux of 0.95 at the midplane. The figure compares the density perturbation from simulations including a single $n=1$ harmonic (i.e. without any toroidal localization/harmonic coupling) with the case with $n=1-5$ coupled harmonics. The width of the perturbation, at half maximum, is reduced by about a factor of 2 due to harmonic coupling.

The simulated density profiles at the outer mid-plane at 3 time slices during one period of the oscillations in Figure $5(a)$ are shown in Figure $7(a)$. Consistent with the boundary displacement mentioned above, the density profiles show a radial displacement of $1.5 \mathrm{~cm}$ within the period. In addition, there is no indication of an island like structure with a local flattening of the profile in the density profiles near the pedestal top, as has been measured in DIII-D in discharges in which RMPs are applied to obtain ELM suppression, although some flattening is observed near the separatrix for the time averaged density profiles, as also measured with the Thomson scattering [27]. Both the magnitude of the density displacement and the density profile shape features are in good agreement with typical observations for QH-modes in DIII-D. This is illustrated in Figure $7(b)$ where the density profiles measured for another QH-mode discharge (shot \#156655) with a fast reflectometer are shown. In this discharge the measured radial displacement due to the EHO is $\sim 1 \mathrm{~cm}$. The simulated density profiles in the presence of a saturated KPM with JOREK for DIII-D are, although not from the same discharge, in good agreement with the profiles from the reflectometer with respect to the amplitude of the displacement and the absence of a local flattening near the pedestal top. 

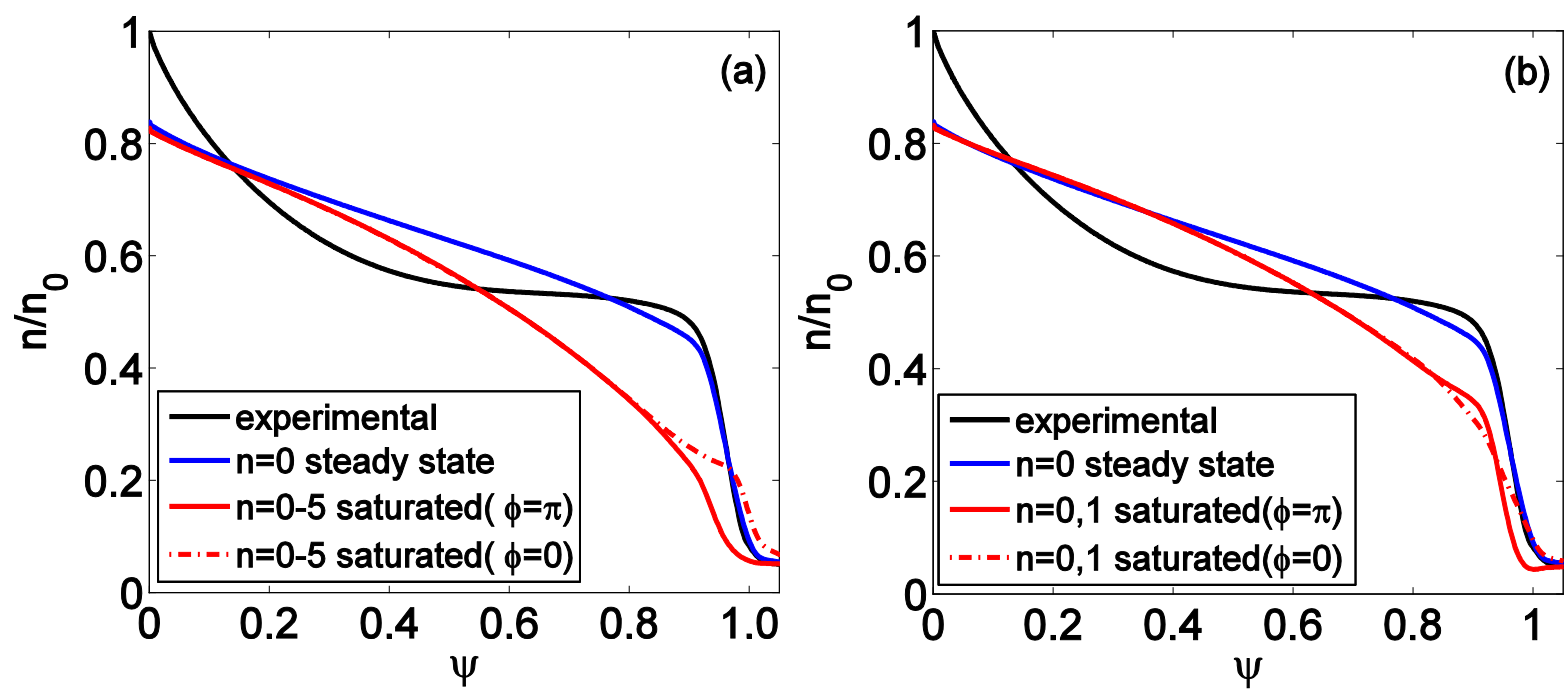

Figure 8. (a) Density profiles for a $\mathrm{n}=[0-5]$ saturated KPM. (b) Density profiles for a $\mathrm{n}=[0,1]$ saturated KPM. The black curves are the initial density profile from experiment, the blue curves show the density profile of the stationary $n=0$ JOREK equilibrium for the shot $\# 145117$. The red curves show the density profiles in the presence of a saturated KPM at $\phi=\pi$ (full curve) and at $\phi=0$ (dashed curve).

Figure 8(a) compares the density profiles at two toroidal phases $(\phi=0, \pi)$ in the presence of saturated $n=[0,5]$ KPM with the stationary JOREK equilibrium state ( $n=0$, blue curve); the experimental density profile (from the time when the EHO is present) is also shown for comparison (black curve). In the simulations it is observed that the presence of a saturated KPM causes a strong additional density loss at the pedestal due to the $\mathrm{E} \times \mathrm{B}$ convection pattern of the saturated kink-peeling mode. In simulations including only the toroidal harmonics $\mathrm{n}=[0,1]$ (see Figure $8(\mathrm{~b})$ ), i.e. not allowing a toroidal localisation of the KPM mode/coupling of multiple harmonics, the stationary density in the presence of the saturated KPM at the top of the pedestal is reduced by $25 \%$ compared with the equilibrium values. In simulations including $n=[0-5]$, the mode becomes more toroidally localised due to coupling of harmonics and the kinetic and magnetic energy of the perturbation increases. In this case the density loss increases and the stationary state density with the saturated KPM at the pedestal top is reduced by 50\% compared to the equilibrium, as shown in Figure 8(a).

On the contrary, the temperature profiles are not significantly affected by the KPM mode. Figure 9 shows temperature profiles at two toroidal phases with saturated KPM for the case with $n=[0-5]$ toroidal harmonics and the stationary state $(n=0)$. Compared to the stationary state, the temperature with KPM has neither significant oscillation at the edge, nor pedestal loss. The physics processes leading to this different behaviour of the density and temperature losses in the presence of a saturated KPM will be discussed in more detail in Section 3. 


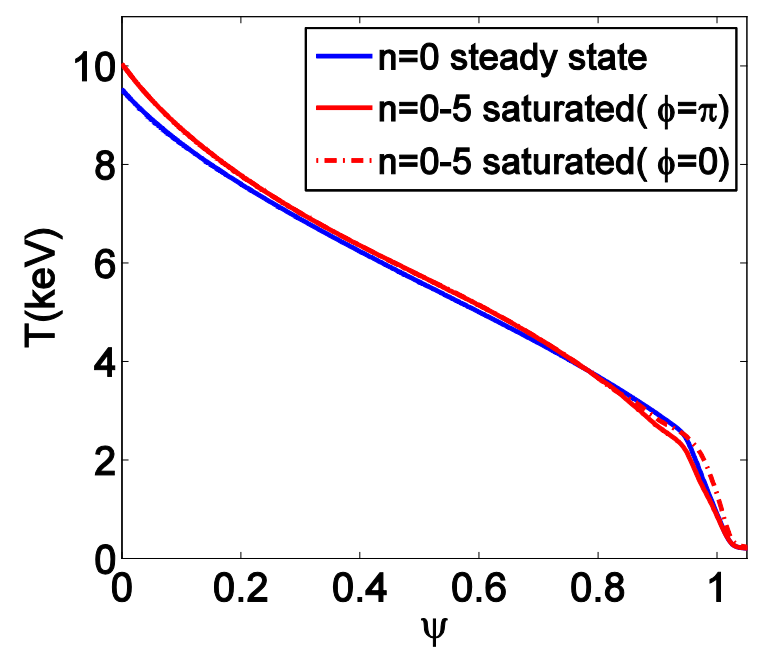

Figure 9. Temperature profiles for a saturated $n=[0-5] \mathrm{KPM}$, the blue curves show the temperature profile of the stationary $n=0$ JOREK equilibrium for the shot $\# 145117$, the red curves show the temperature profiles in the presence of a saturated KPM at $\phi=\pi$ (full curve) and at $\phi=0$ (dashed curve).

The absolute reduction of the pedestal density evaluated by JOREK depends on the assumed amplitude of the total particle source (and the particle transport coefficients chosen as described above), which is $S_{\rho}=1.0 \times 10^{22} \mathrm{~s}^{-1}$ in these simulations. Increasing the total particle source by a factor of 2 and the heat source by a factor of 1.6, and adapting the transport coefficients to keep the same steady state profiles, the reduction of the density at the top of the pedestal is reduced from $\sim 25 \%$ to $\sim 10 \%$ for the $n=[0,1]$ case and from $50 \%$ to $25 \%$ for the $\mathrm{n}=[0-5]$ case. This is due to the fact that the MHD induced particle transport due the $\mathrm{E} \times \mathrm{B}$ flows is not changed significantly by the change in the particle source while the diffusive particle transport is increased by 2. Thus the fraction of the MHD induced transport to the total particle transport is reduced as the magnitude of the particle source increases (and correspondingly the transport coefficients are increased to keep the same equilibrium profiles). Thus for a detailed comparison with experiment on the transport losses induced by the EHO and the saturated KPM in the JOREK simulations, a detailed profile of the particle source in the experiment is required.

Previous JOREK simulations of the plasma response to RMP fields [29] had found that the MHD induced density losses strongly depend on the plasma resistivity with decreasing MHD density losses as the resistivity of the plasma decreases. Therefore we have studied the dependence of the saturated KPM-driven transport versus the value of the resistivity used in the simulations. In the JOREK simulations, the resistivity follows the Spitzer dependence on plasma temperature $\eta(T) \propto T^{-3 / 2}$ [30] with a central resistivity of $\eta_{J}=5 \times 10^{-7}$, corresponding to real resistivity $1.28 \times 10^{-6} \Omega \cdot \mathrm{m}$ in the simulations shown in Figure $3-8$. These simulations 
have thus a Lundquist number $S$ is $6.5 \times 10^{6}$, which is a factor of 100 lower compared to experiment. We have thus scanned the value of the central resistivity resistivity $\eta_{J}$ from $7 \times 10^{-7}$ to $3 \times 10^{-7}$ to quantify its effect on the MHD driven particle transport. Figure $10(a)$ shows the time evolution of the magnetic energy and kinetic energy of simulations including the $n=[0,1]$ harmonics for different values of the resistivity. A reduction of the resistivity $\eta_{J}$ from $7 \times 10^{-7}$ to $3 \times 10^{-7}$ leads to a reduction of the linear growth rate of the unstable KPM by more than a factor of 2 (from $7.2 \times 10^{-3}$ to $2.3 \times 10^{-3}$ ) during its growing phase. The amplitude of the kinetic energy perturbation of the saturated $n=1$ KPM shows a small decrease with the decreasing value of the resistivity, in contrast, the magnetic energy perturbation actually slightly increases. As a consequence the MHD-induced transport due to the KPM is only weakly dependent on the value of the resistivity. This is illustrated more quantitatively in Figure $10(b)$ where the density profiles in the saturated state with for the $n=[0,1]$ KMP for various values of the resistivity are compared with the equilibrium density profiles. From the results in Figure $10(b)$ it is clear that the value of the resistivity within the explored range has only a minor effect on the MHD induced density transport losses and on the resulting density profiles for the phase in which the KPM is saturated, with smaller particle losses being obtained for lower resistivities/lower magnitude of the kinetic energy perturbation. We have also explored the effect of the viscosity on the MHD induced transport during saturated KPMs. However, unlike the resistivity which has a minor effect; the viscosity does not have a noticeable effect either on the KPM growth rate at growing phase or on the amplitude of the saturated KPM or on the density profile during the saturated KPM phase.
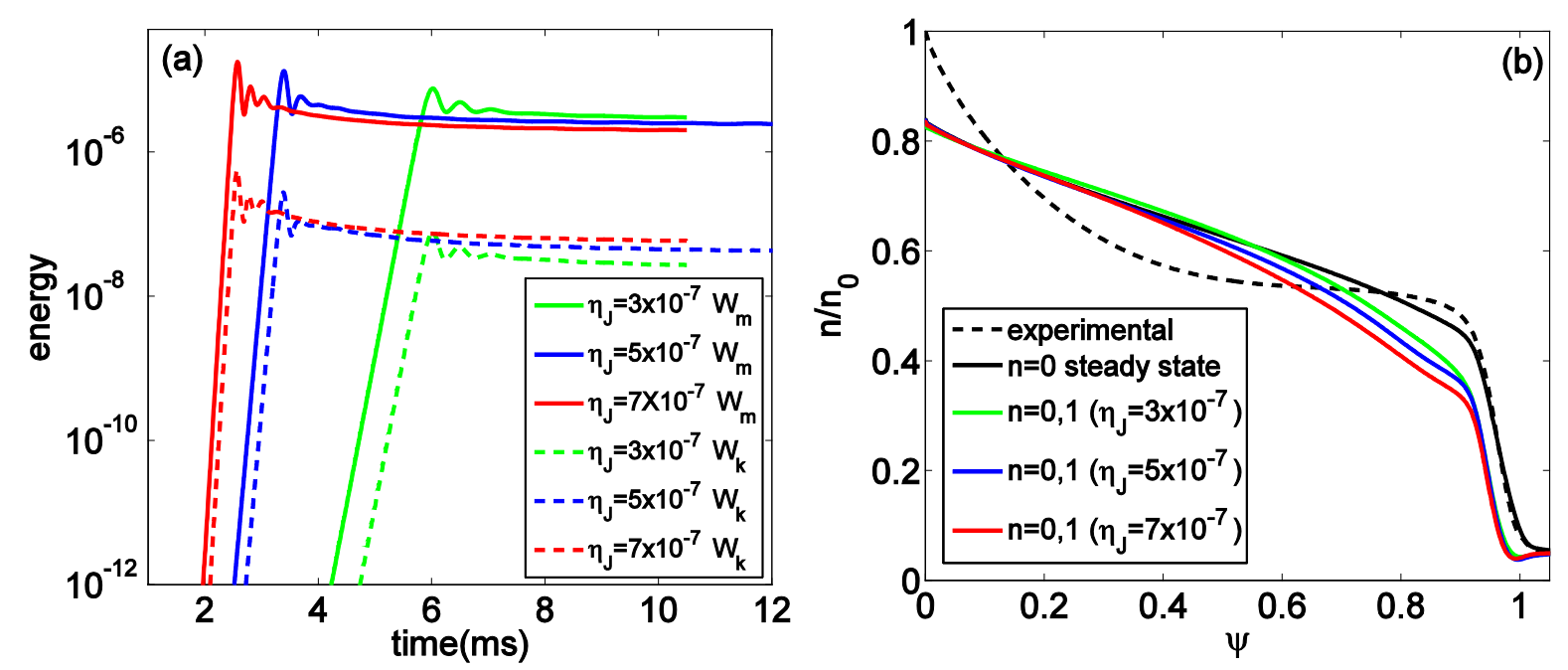

Figure 10. (a) Time evolution of magnetic energy $\left(\mathrm{W}_{\mathrm{m}}\right)$ and kinetic energy $\left(\mathrm{W}_{\mathrm{k}}\right)$ with different resistivities for an $n=1$ KPM for the JOREK simulations of shot \# 145117. (b) Comparison of density profiles with different resistivities for saturated $n=[0,1] \mathrm{KPM}$, initial experiment density profile and $\mathrm{n}=0$ stationary JOREK equilibrium density profile. 

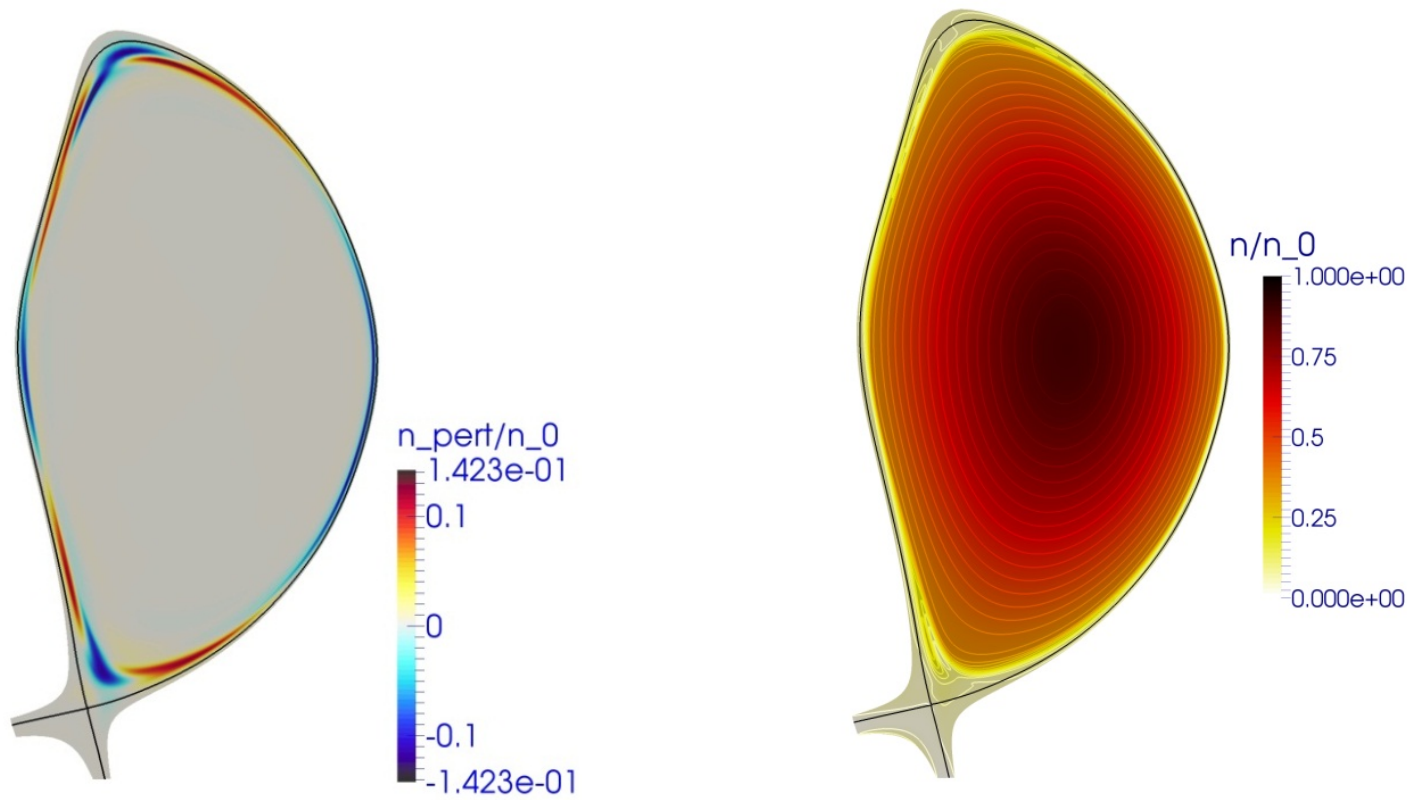

Figure 11. (a) Contour plot of density perturbation in the poloidal plane of an unstable $n=1 \mathrm{KPM}$ at the maximum amplitude in the bursting cycle for \#153440. (b) The density in the poloidal plane and a contour plot of the electric potential with the separatrix in poloidal plane.

JOREK modelling for the DIII-D QH-mode discharge \#153440 (not taking into account the strong counter-rotation in this discharge) shows a very different non-linear behavior compared to discharge \#145117. Simulations of shot \#153440, with similar assumptions as for \#145117, find that for this discharge similar KPMs become unstable to those of \#145117, as shown in Figure 11, but for most conditions no stationary saturated KPM phase is reached; instead a bursting KPM mode is found. This is shown in Figure 12(a) which compares the time evolution of the magnetic energy of the $n=1$ toroidal harmonic for the two discharges \#145117 and \#153440. Instead of the 3-D stationary saturated KPM state found for \#145117 (blue curve), the simulation of \#153440 develops regular bursts of the kink-peeling mode. This bursting behaviour is dependent on both the value of the resistivity and on the value of the edge current density for shot \#153440. Figure 12(b) shows the time evolution of the magnetic energy for the $n=1$ KPM mode with various values of the resistivity and edge current density. As shown in this figure the frequency of bursting decreases with decreasing edge current density. When the initial edge current density is reduced by $20 \%$, (see red curve in Figure 13(a)), the bursting of KPM slows down and its frequency of bursting decreases to about a half of its value with the experimental equilibrium current density profile, as shown in Figure 12(b) (red curve). The bursting behaviour remains as the edge current density is decreased until (when the reduction is larger than $30 \%$ of its initial value) the plasma becomes MHD stable to KPMs. In contrast, when the resistivity is reduced to less than 
$\eta_{J}=4 \times 10^{-7}$, the bursting behavior disappears and the $\mathrm{n}=1$ mode grows with lower growth rate in the linear phase and saturates in a 3-D stationary state at a lower value of the magnetic energy perturbation (see green curve in Figure 12(b)).
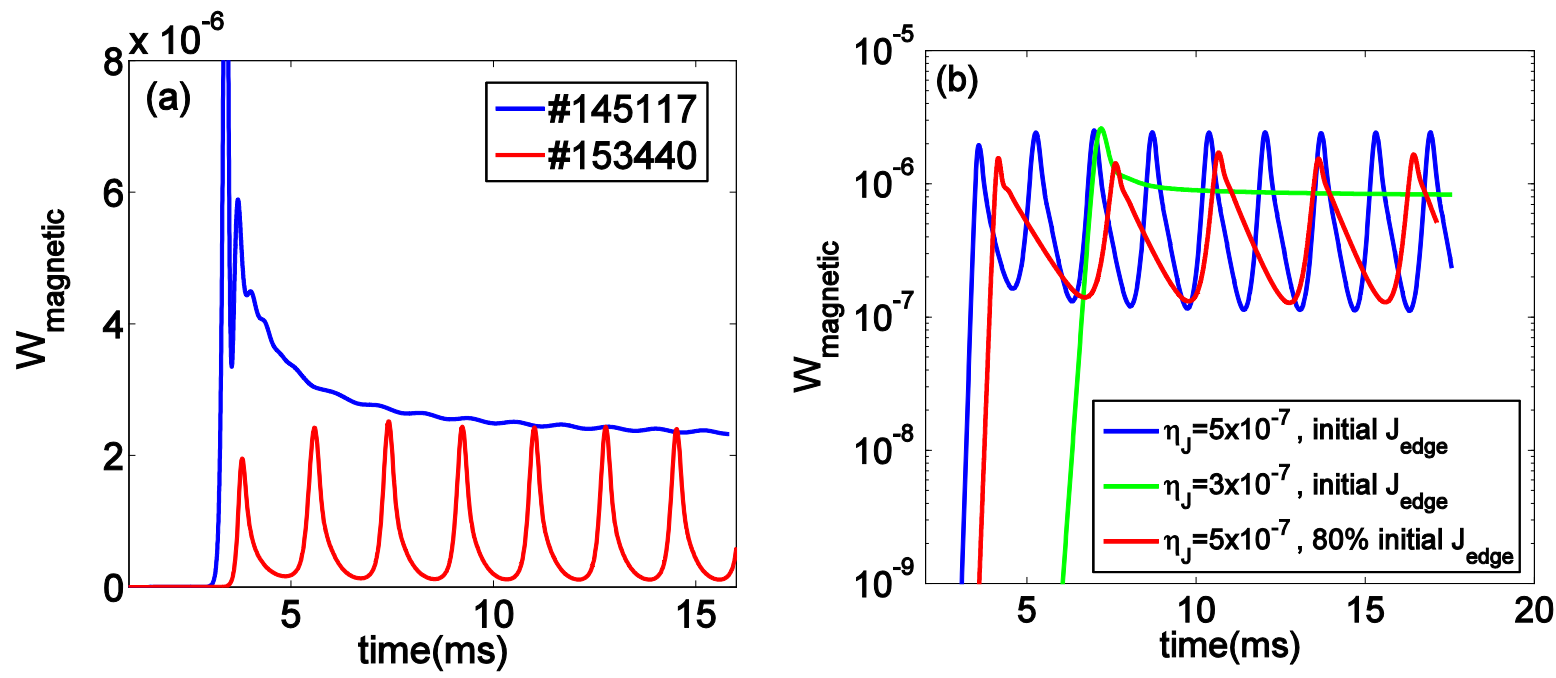

Figure 12. (a) Comparison of the time evolution of the magnetic energy perturbation $(n=1)$ of a 3-D stationary saturated KPM state for \#145117 and a bursting KPM for \#153440. (b) Time evolutions of the magnetic energy of a 3-D stationary bursting KPM $(n=1)$ with various resistivity and edge current density values for \#153440.
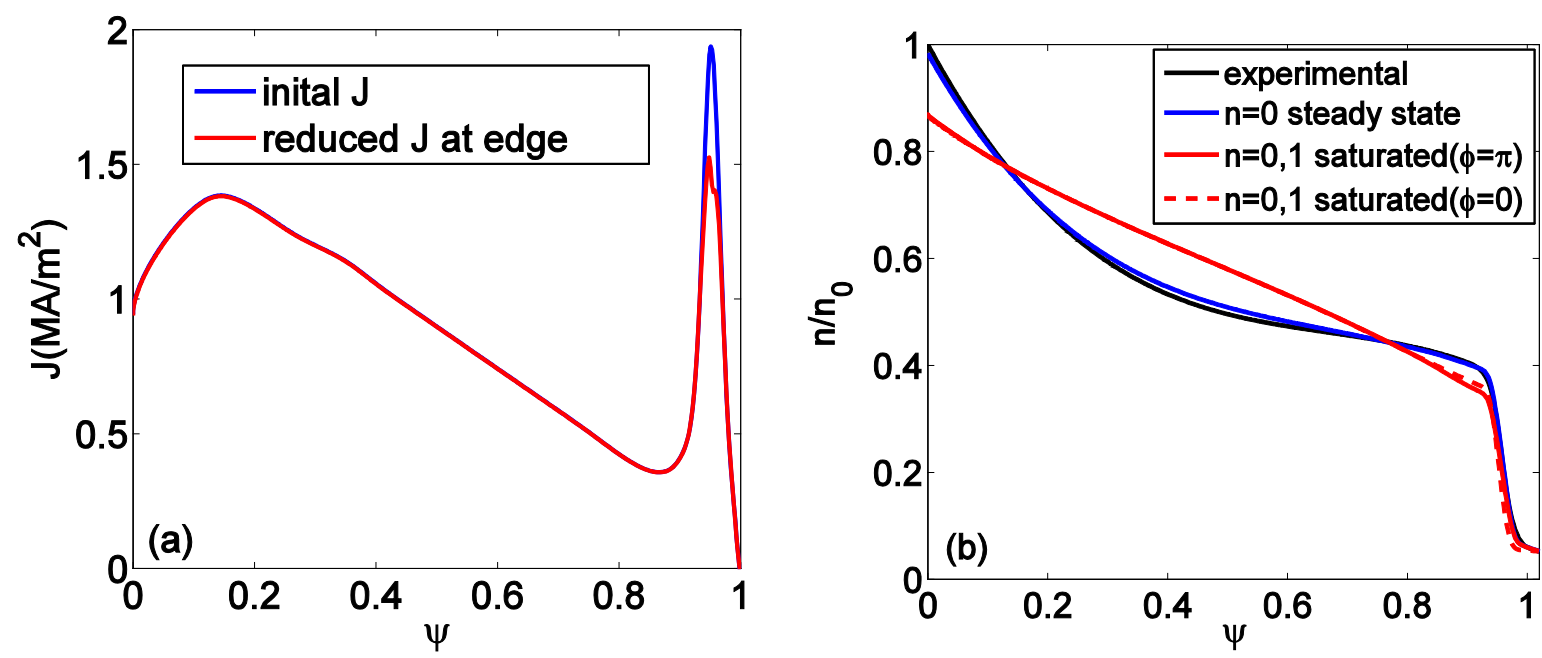

Figure 13. (a) Current density profiles vs normalized $\psi$ for $\# 153440$, the blue curve is the initial current density profile, the red curve is the current density profile with $20 \%$ reduction at the edge. $(b)$ Comparison of density profiles for bursting $n=[0,1]$ KPM in JOREK simulations at the time of maximum amplitude compared to the equilibrium $(n=0)$ profile for $\# 153440$.

There are differences in mode rotation and MHD driven particle transport between the bursting behaviour found for KPMs in shot \#153440 and the stationary saturated KPM state in \#145117. In the 3-D stationary saturated KPM state the mode is continuously rotating with a frequency of $1.6 \mathrm{kHz}$ whereas in the bursting case of \#153440 the KPM only rotates with a similar frequency in the growing phase, once the mode reach the non-linear stage before 
bursting, it stops rotating. The density profile in the bursting state at the time of maximum amplitude has a much smaller oscillation at the edge than that of \#145117, as shown in Figure $13(b)$. The density profile for a bursting $\mathrm{n}=[0,1] \mathrm{KPM}$ at the time of maximum amplitude shows that there is not a significant particle loss (typically the pedestal density decreases by $10 \%$ ) nor a significant oscillation of the boundary when compared with the initial equilibrium pressure profile $(\mathrm{n}=0)$.

One possible reason for the bursting behaviour may be that for shot $\# 153440$ the plasma boundary is much closer to the boundary of the computational domain used in the simulation, where the ideal wall boundary condition is applied, compared to \#145117. This could lead to a larger stabilization of the KPM by induced currents in the wall and thus to the reduction of its amplitude after it has grown to a large amplitude leading to the bursting. Note that in the case of shot \#153440 where 3-D NA fields are not applied in the experiment the large counter rotation together with other rotation terms not included in the modelling here (neoclassical, diamagnetic) may play a role in MHD instability and change the behavior found in the simulations from a bursting to a saturated KPM when included in the simulations. Work is in progress to include these effects (see Section 4).
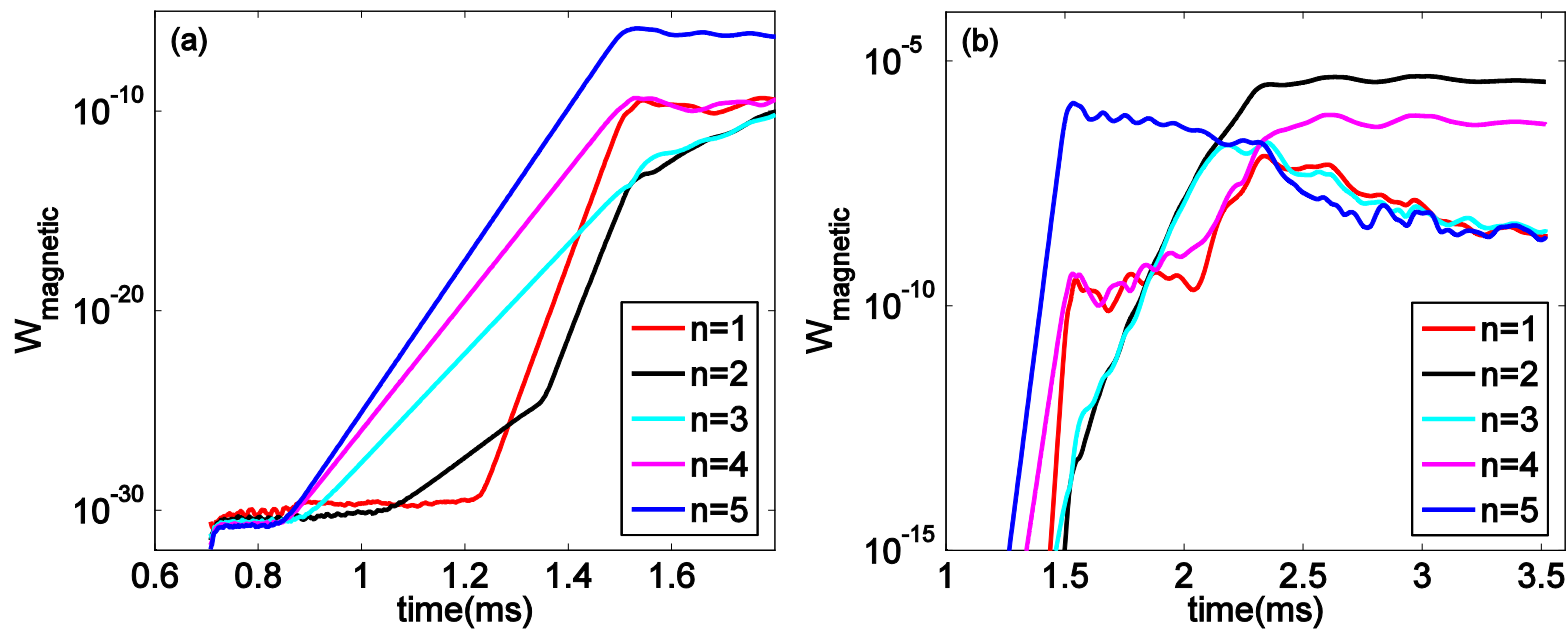

Figure 14. Time evolution of the perturbed magnetic energy of each harmonic for $n=1-5$ kink peeling mode for the JOREK simulations of shot \#153440. (a) Energy evolution in the linear phase, time from $0.6 \mathrm{~ms}$ to $1.7 \mathrm{~ms}$. (b) Energy evolution at the saturated phase, time from $1 \mathrm{~ms}$ to $3.5 \mathrm{~ms}$.

Expanding the number of toroidal harmonics in the simulations of \#153440 to include $n=[0$ 5], a 3D stationary state is obtained, in this case with a dominant toroidal mode number $\mathrm{n}=2$. Figure 14(a) shows that the initial growing phase of the evolution of the magnetic energy is similar for both \#153440 and \#145117. The most unstable mode in the early linear phase is the $n=5$ mode. Due to the non-linear coupling, the modes $n=1$ and $n=2$ develop with a linear growth rate about 10 times larger than their initial linear growth rates. In the non-linear 
phase, the $n=5$ harmonic decays to small amplitude and the $n=2$ and $n=4$ harmonics become dominant as shown in Figure 14(b) (compared to $n=1$ in the case \#145117). The amplitude of the magnetic energy perturbations of the saturated dominant modes $n=[2,4]$ of the KPM for \#153440 are similar to those of $n=[1,2]$ for \#145117 and, correspondingly, the particle losses caused by the $n=[2,4]$ saturated modes are similar to those in $\# 145117$, as shown in Figure 15 while temperature losses remain very small. It is interesting to note that to obtain a toroidal localization, which is the lower energy state of the saturated KPMs obtained in JOREK modelling, the harmonics which are a multiple of the dominant mode need to be locked in phase. In the case of a dominant $n=2$ mode, only the even modes contribute to the toroidal localization of the KPM mode and the odd modes decay to a small amplitude. This explains the fact that for the simulations of $\# 153440$ with $n=[0-5]$ harmonics only the $n=2$ and $n=4$ harmonics saturate at high magnetic energy perturbation amplitude.
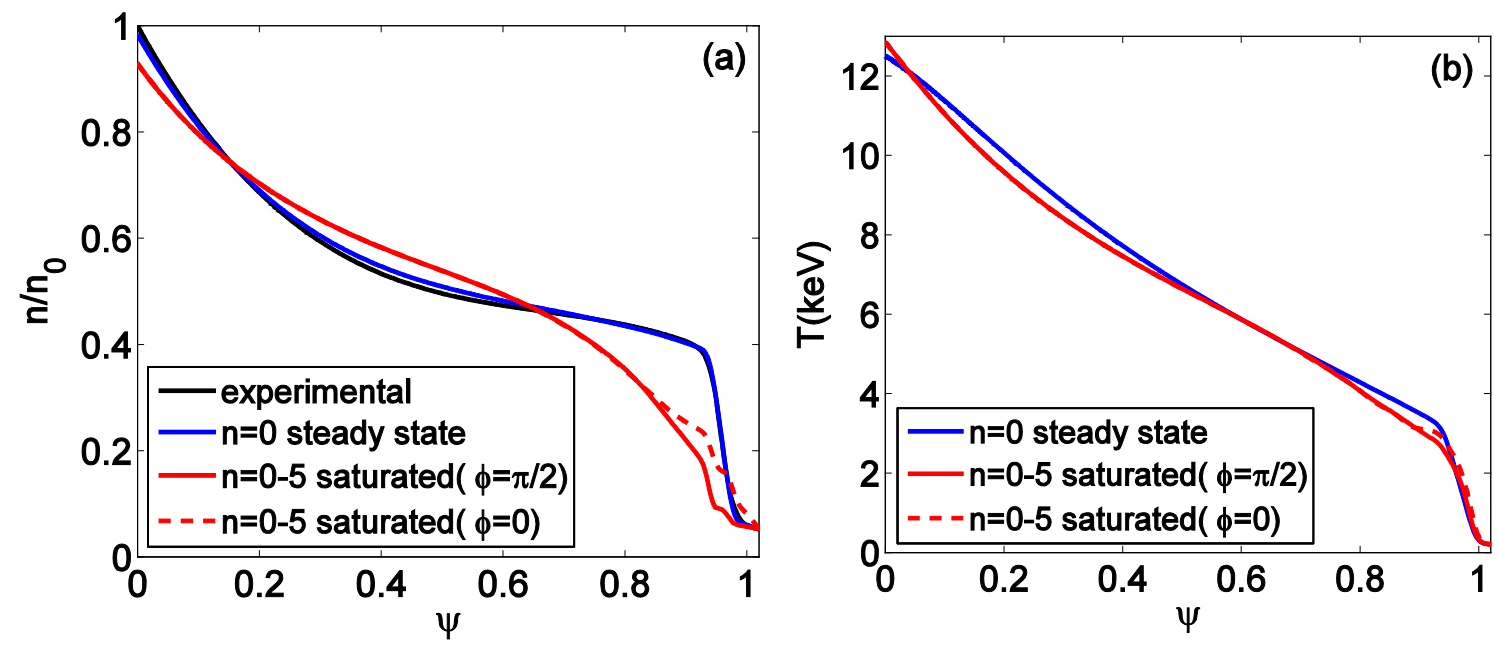

Figure 15. (a) Density profiles for the $\mathrm{n}=[0-5]$ saturated KPM. (b) Temperature profiles for a $\mathrm{n}=[0-5]$ saturated KPM. The black curves are the initial density profile from experiment, the blue curves show the density profile of the stationary $n=0$ JOREK equilibrium for the shot $\# 153440$. The red curves show the density profiles in the presence of a saturated KPM at $\phi=\pi / 2$ (full curve) and at $\phi=0$ (dashed curve).

The simulations for both DIII-D QH-mode plasmas show that KPMs can saturate even in the absence of a significant rotation in the simulations. This indicates that the interaction of the kink-peeling mode with a resistive wall is not an essential ingredient for its saturation for these plasma conditions in the simulations. A possible reason for the mode saturation is the formation of islands and an ergodic layer in the edge of the plasma, which has been found in the simulations of both QH-mode plasmas. The Poincare plot of magnetic field lines for a saturated n=1-5 KPM at the edge plasma of \#145117 in Figure 16(a) shows these features.

A strongly ergodic field would lead to a large energy loss due to the large parallel conduction losses, as is found in simulations of plasmas with resonant magnetic field perturbations for 
ELM control. However, in these QH-mode simulations the temperature losses are small. To estimate the heat transport due the magnetic field perturbations, an upper estimate for the effective perpendicular heat diffusion coefficient (assuming free-streaming electrons) has been calculated following [31]:

$$
\left\langle\kappa_{\perp} \operatorname{erg}\right\rangle=\frac{\left\langle(\Delta \psi)^{2}\right\rangle}{L_{\|}} v_{t h, e}
$$

Figure 16a shows a Poincare plot with the color scale indicating the local perpendicular heat diffusion coefficient. Averaging over flux surfaces gives a maximum value for the estimated effective radial transport due to the ergodic field of $\kappa_{\perp}$ erg $\sim 0.5 \mathrm{~m}^{2} / \mathrm{s}$ in the pedestal region, which is comparable to the value of $\kappa_{\perp \text { ped }}$ used in the simulations $\left(\kappa_{\perp \text { ped }}=0.5 \mathrm{~m}^{2} \mathrm{~s}^{-1}\right)$. However, in the non-linear MHD simulations presented here, the parallel conduction is reduced by a factor of 25 with respect to its real Spitzer-Harm value for numerical reasons. Therefore, the expected ergodic perpendicular heat transport in these QH-mode simulations is very low, much lower than the upper estimate from Eq. 8, of the order of $0.02 \mathrm{~m}^{2} / \mathrm{s}$. This is consistent with the very small change to the temperature profile found in the simulations. This could change if larger values of parallel conduction, closer to the real Spitzer-Harm value, would be used in the simulations. On the basis of the arguments above a change of gradient of at most a factor of $\sim 2$ would be expected in the pedestal in that case, as $\kappa_{\perp p e d}$ eff $=\kappa_{\perp p e d}+\kappa_{\perp}^{e r g} \approx 2 \kappa_{\perp p e d}$. Independently of this, the length of the field lines, indicated by the color scale in the Poincare plot Figure 16(b), shows that although the field is ergodic when the KPM is saturated, the field lines do not have a short connection length to the divertor targets; i.e. their radial excursions remain limited and within the confined plasma. Thus, although ergodic transport could potentially affect the shape of the temperature profiles in the pedestal region for larger values of the parallel conductivity when the KPM is saturated, the direct losses on open field lines connecting the plasma facing components to the confined plasma will always remain small with a saturated KPM in the simulations. On the other hand, the corresponding plot of the density in the poloidal plane in Figure 16(c) shows that the density perturbation follows the contour lines of the electric potential (i.e. the $\mathrm{E} \times \mathrm{B}$ flows) which are not necessarily aligned with the magnetic field lines. The $\mathrm{E} \times \mathrm{B}$ flow lines do cross the plasma boundary and leads to the modelled particle losses. Further work is in progress to understand the precise mechanisms that may lead to KPM saturation in the presence of an ergodic layer. 


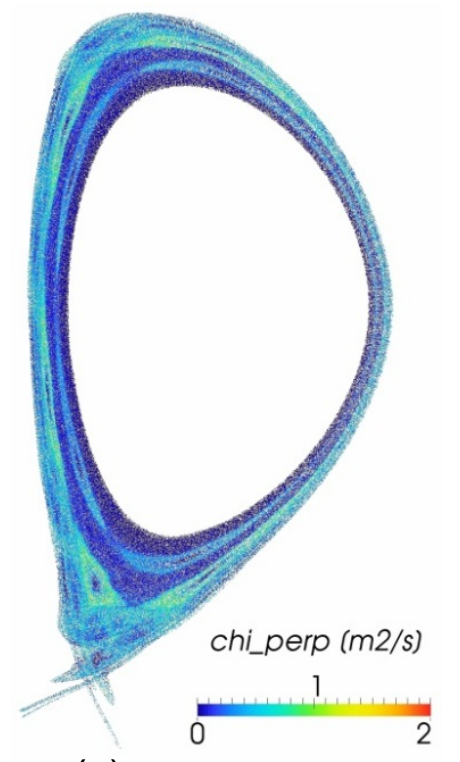

(a)

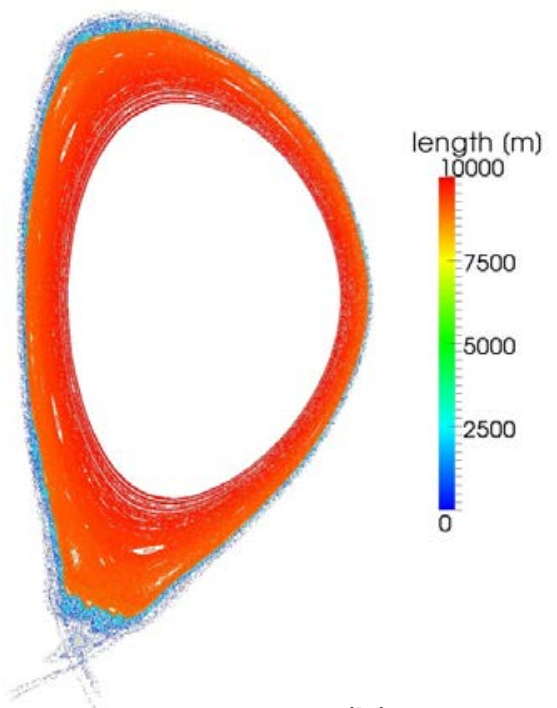

(b)

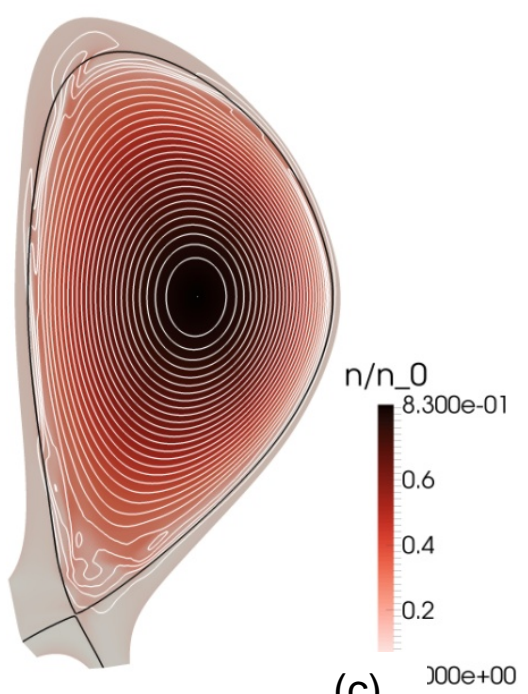

(c)

Figure 16. Poincare plot of magnetic field lines for the saturated $n=1-5$ KPM of $\# 145117$, the color scale indicates the estimated heat transport due to the ergodicity $(a)$ and the connection length $(b)$. $(c)$ shows the density in the poloidal plane and a contour plot of the electric potential with the separatrix in poloidal plane.

\section{Effect of parallel equilibrium rotation on the QH-mode non-linear evolution}

Recent investigations [12, 25] indicate that the EHO is possibly driven by edge rotation and rotation shear destabilizing KPMs. In order to understand the effect of edge rotation in QH modes edge stability, parallel rotation, as an approximation of the toroidal rotation has been included in the JOREK simulations by utilizing a profile for the parallel rotation similar to the toroidal rotation measured experimentally. Figure 17(a) shows the 2-D stationary equilibrium including parallel rotation, in the plasma and in the scrape-off layer region and divertor for \#145117. The parallel rotation at the top of the pedestal in the simulations is about $20 \mathrm{~km} / \mathrm{s}$, which is similar to that measured. The time evolution of the magnetic energy for the modes $n=1-5$ with parallel rotation is shown in Figure 17(b) for $\# 145117$. Compared to the case without parallel rotation for the same shot, the parallel rotation profile (with a strong rotation shear at the pedestal) at this amplitude level does not significantly change the linear growth rates or the non-linear evolution. The saturation levels are comparable with and without parallel rotation (compare Figure $17(b)$ with Figure $3(b)$ ), although the case with rotation exhibits a more irregular time behaviour. The density (and pressure) at the top of the pedestal has the same reduction and the EHO frequency obtained is also similar for both cases. On this basis it appears that the parallel rotation and/or its rotational shear on its own does not play a major role in the destabilization and saturation of the KPMs leading to the EHO in the DIII-D QH-modes modelled. 

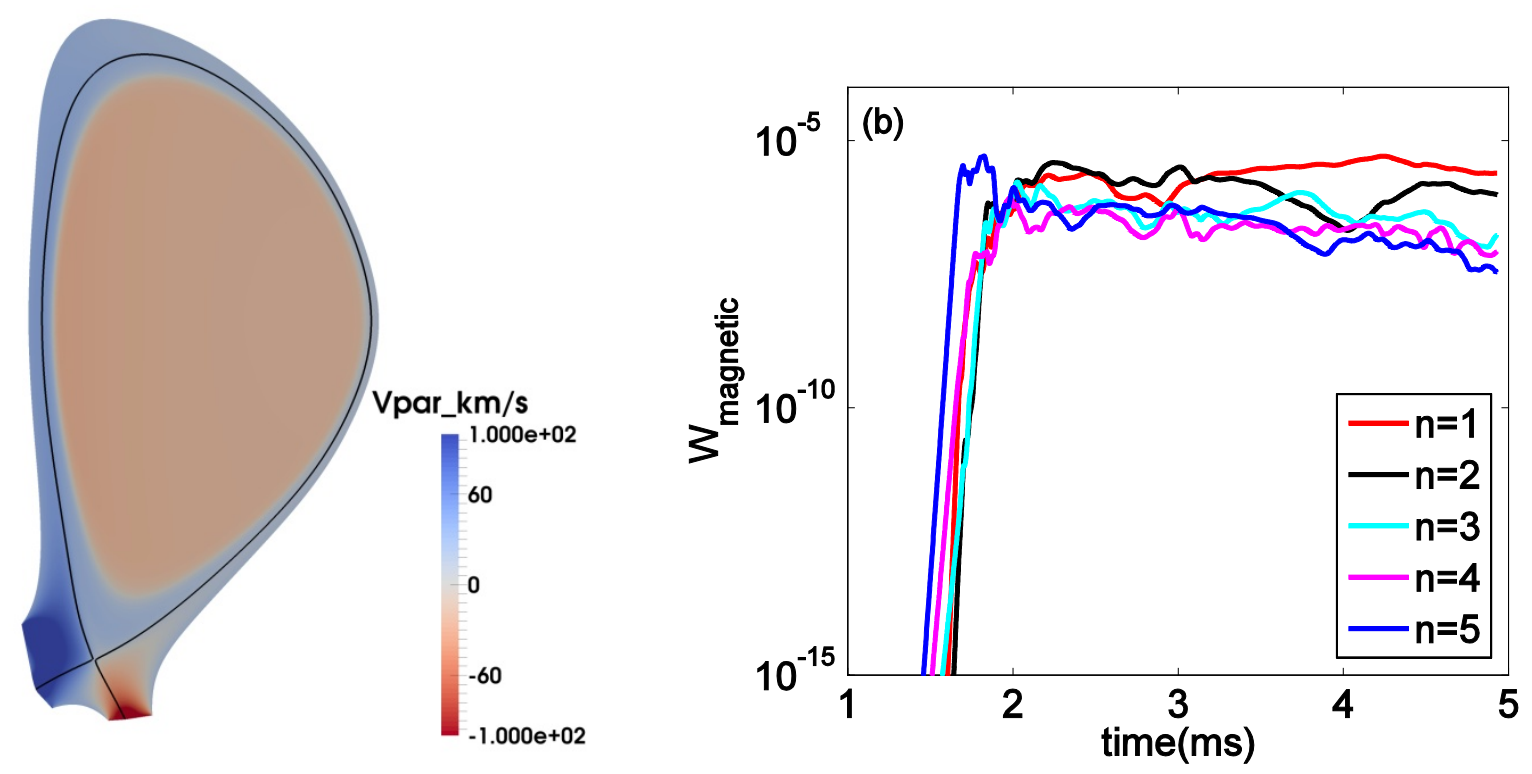

Figure 17. (a) 2-D stationary equilibrium in the poloidal plane with parallel flow. (b) Time evolution of magnetic energy for n=1-5 KPM of \#145117 with parallel equilibrium rotation.

\section{Effect of a resistive wall on the QH-mode non-linear evolution}

The influence of a realistic resistive wall in these DIII-D simulations has been studied with the coupled codes JOREK and STARWALL [20]. The STARWALL code calculates the response from the vacuum and the time evolution of the induced eddy currents in the resistive wall, using a thin wall approximation. This dynamic response is used as a boundary condition on the poloidal flux evolution in the JOREK code.

Figure 18(a) shows the plasma flux perturbation and the electric potential in the resistive wall during the saturated state of an $n=[0,1]$ KPM for \#145117. Figure 18(b) shows the time evolution of the magnetic energy of the KPM mode for an ideal wall without plasma rotation and for a resistive wall for a range parallel rotation speeds at the plasma pedestal from $80 \mathrm{~km} / \mathrm{s}$ to $80 \mathrm{~km} / \mathrm{s}$. As shown in this figure the effects of a resistive wall and of toroidal rotation with a resistive wall on KPM instability and saturation are very moderate. With a resistive wall there is a small decrease in the linear growth rate of the KPM by about $20 \%$ when the toroidal rotation increases from 0 to $80 \mathrm{~km} / \mathrm{s}$. In contrast, the linear growth rate of the KPM with counter parallel rotation is very close to the ideal wall case. The saturation level is also modified by positive parallel rotation in the resistive wall case, with the highest saturation levels being found for the highest positive parallel rotation speeds. As for the linear growth rate of the KPM, its saturation level is not modified by rotation speed for the cases with counter parallel rotation and it is similar to the ideal wall case with no rotation. It should be noted that for all cases the effects of toroidal rotation are small with a resistive wall and, in 
fact, the the density loss at the pedestal is reduced from $25 \%$ to below $20 \%$ when parallel rotation increases from 0 to $80 \mathrm{~km} / \mathrm{s}$, despite the increase of saturated magnetic energy of the KMP. This shows that indeed the inclusion of a resistive wall and plasma rotation has an effect on the non-linear KPM evolution but that this effect is small in these simulations of the QH-mode conditions in DIII-D.
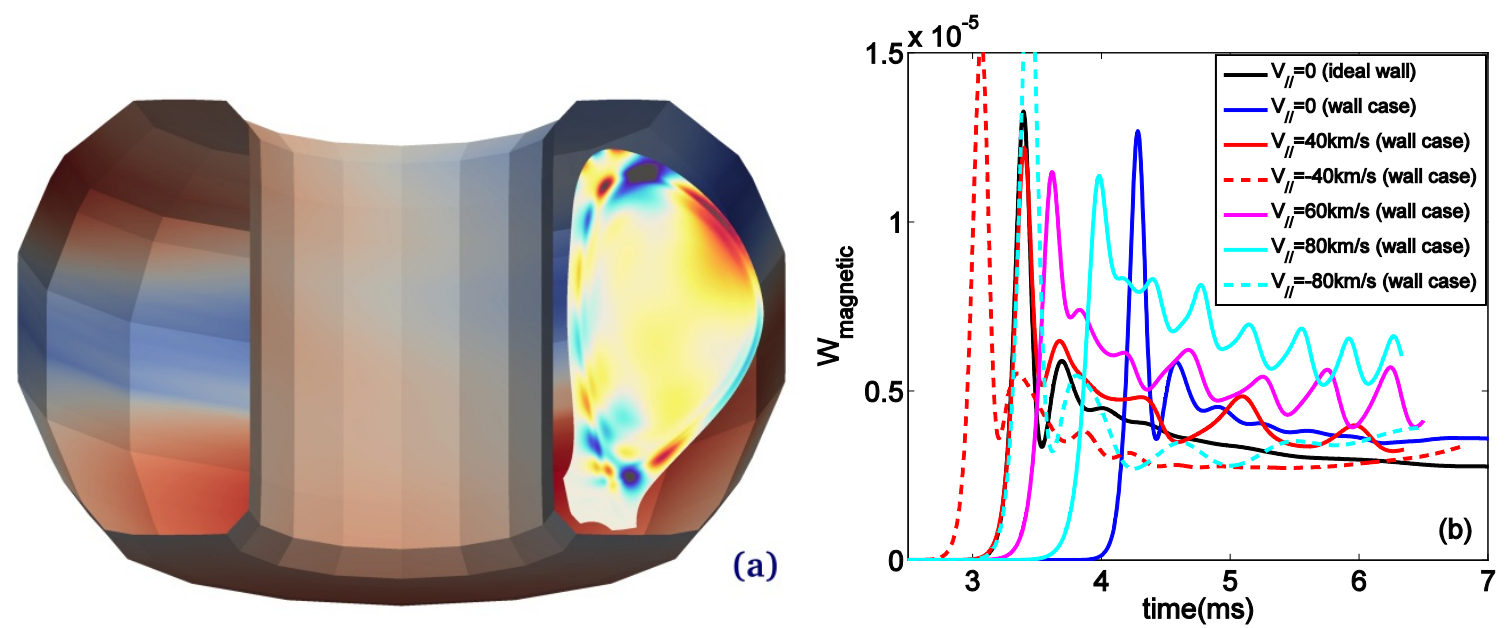

Figure 18. (a) The perturbed flux and wall potential on the resistive wall of a saturated $n=1$ KPM for \#145117. (b) The $(\mathrm{n}=1)$ magnetic energy for ideal wall and a scan of the assumed parallel rotation speed at the top of the pedestal for the resistive wall.

It is also important to note that, for the cases with zero parallel rotation speed, the KPM saturation level is very similar for the case with an ideal wall and a resistive wall. In both cases the saturated KPM rotates with similar frequency in the poloidal plane, and the resulting density loss at pedestal is also comparable. Thus with a resistive wall the saturation of the KPM does not require a finite toroidal rotation, as found with an ideal wall.

\section{Conclusions}

Both linear and nonlinear MHD simulations for two DIII-D QH-mode discharges have been performed with the MISHKA and JOREK code respectively. The linear MHD peelingballooning stability study shows that these QH-mode plasmas are close to the kink/peeling stability boundary, confirming earlier studies $[12,25]$, with the kink/peeling stable boundary being sensitive to the value of the separatrix current density. In the nonlinear MHD simulations of DIII-D QH-mode plasmas, kink/peeling modes have been found to grow exponentially in the linear phase and then non-linearly saturate into a new 3-D stationary state. It is found that the dominant toroidal mode number in the 3-D stationary state is not determined by the most unstable linear instability but that the low-n $(n=1,2)$ modes in the 3D state are excited by the non-linear coupling of the medium-n harmonics. The saturated KPM mode leads to oscillations in the edge magnetic field, position of the plasma boundary 
and associated plasma parameters (density in particular) with the typical multi-harmonic content associated with the EHO in QH-mode plasmas observed experimentally in magnetics and fast reflectometer measurements. In the non-linear simulations, the $\mathrm{E} \times \mathrm{B}$ flows due to the saturated kink-peeling mode lead to a significant particle loss channel and, associated with it, to a decrease of the edge plasma density, which is in good agreement with typical observations in DIII-D QH-mode plasmas. On the basis of the non-linear MHD modelling results presented in this paper we can conclude that the experimental behaviour observed for the EHO is in good agreement with that modelled for a saturated kink/peeling mode driven unstable by the large edge current density in these plasmas, as previously conjectured.

The main issue which remains to be understood is that of the mechanism leading to the saturation of the mode and whether this mode remains saturated in a stationary fashion or if it is subject to relaxations (bursty behaviour). In our simulations we find that this is affected by resistivity of the plasma, proximity to a conducting ideal wall and where edge ergodization by the mode itself could potentially play a role. In general the effects of parallel rotation/rotation shear on the saturation of the KPM appear to be small even when we consider a realistic resistive wall, which is contrary to current experimental evidence. This could be due to the fact that the present simulations only include parallel rotation effects and that, when self-consistent simulations including neoclassical poloidal velocities and diamagnetic effects to properly reproduce the radial electric field, then the edge non-linear MHD stability picture may be changed. This will be subject to further studies to be carried out in conjunction with a realistic resistive wall.

An important question that remains to be addressed is which are the physics mechanisms and which are the relevant parameters determining whether the plasma develops into an ELMy Hmode, dominated by the non-linear evolution of ballooning modes or into a QH-mode regime in which the relevant modes are low-n kink-peeling modes.

Ultimately the identification of the physics mechanisms that lead to the saturation of the KPM and the EHO in DIII-D QH-mode will allow us to evaluate the possibility of high fusion performance ITER operation in this operational mode taking into account the specific characteristics of ITER plasmas and tokamak (low resistivity/low collisionality plasma, low toroidal rotation, highly conducting vessel at $\mathrm{r}_{\text {wall }} / \mathrm{a}=1.4$, etc.).

This work was supported in part by the US Department of Energy under DE-FC02-04ER54698 and DE-AC03-09CH11466. The views and opinions expressed herein do not necessarily reflect those of the ITER Organization. 


\section{References}

[1] Loarte A. et al 2003 Plasma Phys. Control. Fusion 451549

[2] Bazylev B. et al 2007 Phys. Scr. T128 229-33

[3] Loarte A. et al 2007 Nucl. Fusion 47 S203

[4] Lang P. T. et al 2008 Nucl. Fusion 48095007

[5] Evans T.E. et al 2013 Nucl. Fusion 53093029

[6] Degeling A.W. et al 2003 Plasma Phys. Control. Fusion 451637

[7] Burrell K.H. et al 1999 Bull. Am. Phys. Soc. 44127

[8] Burrell K.H. et al 2001 Phys. Plasmas 82153

[9] Suttrop W. et al 2005 Nucl. Fusion 45721

[10] Sakamoto Y. et al 2004 Plasma Phys. Control. Fusion 46 A299

[11] Oyama N. et al 2005 Nucl. Fusion 45871

[12] Garofalo A.M. et al 2011 Nucl. Fus. 51083018

[13] Solomon W. et al 2013 Bull. Am. Phys. Soc. 58

[14] Snyder P.B. et al 2007 Nucl. Fusion 47961

[15] Huysmans G.T.A. et al 2007 Nucl. Fus. 47

[16] Huysmans G.T.A. et al 2009 Plasma Phys. Control. Fusion 51124012

[17] Lao, L.L., et al., Nucl. Fusion 30, 1035 (1990).

[18] Huysmans G.T.A. et al 2001 Phys. Plasmas 84292

[19] Snyder P.B. et al. 2002 Phys. Plasmas 92037

[20] Hoelzl M. et al. 2012 JPCS 401012010

[21] Stangeby P.C. et al. 1995 Phys. Plasmas 2 (3) 707

[22] Czarny O. and Huysmans G. 2008 J. Comput. Phys. 227 7423-45

[23] Henon P., et al 2002 Parallel Computing 28 (2) 301-321

[24] Huysmans G.T.A. 2005 Plasma Phys. Control. Fusion 47 B165-78

[25] Burrell K.H. et al. 2013 Nucl. Fusion 53073038

[26] Suttrop W. et al. 2005 Nucl. Fusion 45 721-730

[27] Garofalo, A, et al., submitted to Physics of Plasmas.

[28] Zeng L. (UCLA), private communication.

[29] Nardon E. et al 2010 Nucl. Fusion 50034002

[30] Nardon E. et al 2007 Phys. Plasmas 14092501 
[31] Finken K.H. et al. 2005 Schriften des Forschungszentrums Jülich Reihe Energietechnik / Energy Technology, Vol. 45, ISBN 3-89336-418-8, Editor Forschungszentrum Jülich $\mathrm{GmbH}$. 